\title{
Relativistic Anyon Beam: Construction and Properties
}

\author{
Joydeep Majhi, Subir Ghosh, and Santanu K. Maiti* \\ Physics and Applied Mathematics Unit, Indian Statistical Institute, 203 Barrackpore Trunk Road, Kolkata-700 108, India
}

(Received 13 February 2019; revised manuscript received 9 August 2019; published 17 October 2019)

\begin{abstract}
Motivated by recent interest in photon and electron vortex beams, we propose the construction of a relativistic anyon beam. Following Jackiw and Nair [R. Jackiw and V. P. Nair, Phys. Rev. D 43, 1933 (1991).] we derive an explicit form of the relativistic plane wave solution of a single anyon. Subsequently we construct the planar anyon beam by superposing these solutions. Explicit expressions for the conserved anyon current are derived. Finally, we provide expressions for the anyon beam current using the superposed waves and discuss its properties. We also comment on the possibility of laboratory construction of an anyon beam.
\end{abstract}

DOI: 10.1103/PhysRevLett.123.164801

We propose construction of a relativistic beam of anyons in a plane. Anyons are planar excitations with arbitrary spin and statistics. The procedure is similar to threedimensional (3D) optical (spin 1) and electron (spin 1/2) vortex beams.

Vortex beams, of recent interest, carrying intrinsic orbital angular momentum are nondiffracting wave packets in motion. In 3D, vortex beams for photons and electrons were proposed by Durnin et al. [1] and by Bliokh et al. [2], respectively (see [3]). Properties and experimental signatures of twisted optical and electron vortex beams were studied respectively in [4] (for spinless electron [2]) and [5] (spinor electron [2]). Wave packets were constructed out of free plane wave, orbital angular momentum solutions having vorticity. In 3D, the nontrivial Lie algebra of rotation generators along with finite-dimensional representations of angular momentum eigenstates restrict the angular momentum to quantize in units of $\hbar / 2$. The absence of such an algebra in two dimensions (2D) allows excitations (anyons [6,7]) with arbitrary spin and statistics with infinite-dimensional representations. Here, we construct a directed transversely localized anyon wave packet or wave beam in 2D.

Geometry imposes a qualitative difference between monoenergetic wave packet and wave beam both in 3D and $2 \mathrm{D}$. In $3 \mathrm{D}$ the wave packet is described by three discrete quantum numbers as it is localized in three directions whereas ideally an energy dispersionless wave packet where wave components having identical momentum along the direction of propagation will be completely delocalized along that direction and will constitute a

Published by the American Physical Society under the terms of the Creative Commons Attribution 4.0 International license. Further distribution of this work must maintain attribution to the author(s) and the published article's title, journal citation, and DOI. Funded by SCOAP ${ }^{3}$. beam $[1,2]$. But, in 2D a monoenergetic wave beam will necessary have dispersion in momentum along propagation direction and hence cannot be completely delocalized. The delocalization along propagation direction will be more for a narrower angle of superposition.

Apart from earlier interest [6], graphene also involves anyons [8]. Non-Abelian anyons [9] are touted as theoretical building blocks for topological fault-tolerant quantum computers [10]. An exact chiral spin liquid with non-Abelian anyons has been reported [11]. The direct observational status of anyons has shown promising development [12].

The minimal field theoretic and relativistic model of a single anyon was constructed by Jackiw and Nair (JN) [13]. $\mathrm{JN}$ anyon is the most suitable one that serves our purpose since, being first order in spacetime derivatives, it closely resembles the Dirac equation used by [2]. A major difference is that unitary representation for an arbitrary spin JN anyon requires an infinite component wave function [14] (to maintain covariance), along with subsidiary constraints that restrict the number of independent components to a single one [13].

We propose (i) an explicit form of the single anyon solution of the JN anyon equation [13] (which is a new result), (ii) an anyon conserved current (again a new result), (iii) an anyon wave packet in 2D, (iv) an anyon wave packet in the conserved current that constitutes the anyon beam, (iv) anyon charge and current densities for the anyon beam numerically since closed analytic expressions could not be obtained, (v) a possible laboratory setup for observing anyon beams, and (vi) finally, future prospects. The new results [(i) and (ii)] lead us to our principal outputs [(iii) and (iv) and hopefully (v)].

(i) Jackiw-Nair anyon equation and its solution: We start with a familiar system, free spin one particle in $2+1$ dimensions [13]. The dynamical equation in coordinate and momentum space $\left(i \partial_{a}=p_{a}\right)$ is given by 


$$
\partial_{a} \epsilon^{a b c} F_{c} \pm m F^{b}=0 ; \quad(p j)_{b}^{a} F^{b}+m s F^{a}=0 .
$$

The solution of the three-vector $F^{a}, a=0,1,2$ [we use Minkowski metric $\left.\eta_{\mu \nu}=\operatorname{diag}(1,-1,-1)\right]$ is given by

$$
F^{a}(p)=\frac{1}{\sqrt{2}}\left[\left[\begin{array}{l}
0 \\
1 \\
i
\end{array}\right]+\frac{p^{x}+i p^{y}}{m(E+m)}\left[\begin{array}{c}
E+m \\
p^{x} \\
p^{y}
\end{array}\right]\right] \sqrt{\frac{m}{E}} .
$$

The same $F^{a}$ can also be expressed as a Lorentz boosted form,

$$
F^{a}(p)=B_{b}^{a}(p) N_{c}^{b} F_{0}^{c}(p), \quad N_{c}^{b}=\frac{1}{\sqrt{2}}\left[\begin{array}{ccc}
\sqrt{2} & 0 & 0 \\
0 & 1 & i \\
0 & -i & i
\end{array}\right],
$$

where the boost is expressed by the generators $j^{a}$,

$$
\begin{aligned}
B(p) & =e^{i \Omega_{a}(p) j^{a} ;}[B(p)]_{b}^{a}=\left[B^{-1}(p)\right]_{b}^{a} \\
& =\delta_{b}^{a}-\frac{\left(p^{a}+\eta^{a} m\right)\left(p_{b}+\eta_{b} m\right)}{m(p \eta+m)}+\frac{2 p^{a} \eta_{b}}{m},
\end{aligned}
$$

with $\eta_{a}=(1,0,0)$. It is straightforward to check that $F^{a}$ describes a spin one particle $(s=1)$ of mass $m$.

This construction has been extended in an elegant way to the $\mathrm{JN}$ anyon equation [13] to describe an anyon of arbitrary spin $s=1-\lambda$, whose dynamics in momentum space is given by,

$$
P \cdot(K+j)_{a n a^{\prime} n^{\prime}} f_{n^{\prime}}^{a^{\prime}}+m s f_{a n}=0, \quad\left(D_{a} f^{a}\right)_{n}=0,
$$

$\left(D_{n n^{\prime}}^{a}=\epsilon_{b c}^{a} P^{b} K_{n n^{\prime}}^{c}\right)$ where the second equation is the subsidiary (constraint) relation. For $\lambda=0$ the anyon reduces to the spin one model discussed earlier [13]. The actions of $j^{a}, K^{a}$ are given by

$$
\begin{aligned}
P K_{a n a^{\prime} n^{\prime}} & =P K_{n n^{\prime}} \delta_{a a^{\prime}}, \quad P j_{a n a^{\prime} n^{\prime}}=P j_{a a^{\prime}} \delta_{n n^{\prime}} ; \\
K_{n n^{\prime}}^{a} & =\left\langle\lambda, n\left|K^{a}\right| \lambda, n^{\prime}\right\rangle, \quad\left(j^{a}\right)_{a^{\prime} a^{\prime \prime}}=i \epsilon_{a^{\prime} a^{\prime \prime}}^{a}
\end{aligned}
$$

Generalizing the spin 1 case (3), the free anyon solution is formally given by [13]

$$
f_{n}^{a( \pm)}(p)=B_{n 0}(p) B_{b}^{a}(p) N_{c}^{b} f^{c( \pm)}(p),
$$

where $B_{n n^{\prime}}(p), B_{b}^{a}(p)$ are the spin $\lambda$ and spin 1 representations of the boost transformation respectively and $N_{c}^{b}$ is the same numerical matrix as in (3).

Exploiting coherent state formalism for $S U(1,1)[15,16]$ along with the $S U(1,1) \sim S O(2,1)$ connection, we have constructed an explicit form of $B_{n 0}(p)$ [in a matrix representation of $K^{a}$ [13] (see, e.g., [17])]. Computational details are in Supplemental Material A [18]. The free anyon solution is

$$
\begin{aligned}
f_{n}^{a+}= & \left(\frac{2 m}{E+m}\right)^{\lambda} \sqrt{\frac{\Gamma(2 \lambda+n)}{n ! \Gamma(2 \lambda)}}\left(\frac{p^{x}+i p^{y}}{E+m}\right)^{n} \\
& \times F^{a}(p) e^{-i p x},
\end{aligned}
$$

where $F^{a}(p)$ is the same as the spin 1 case defined in (2). This is our primary result that we exploit to construct the wave packet and subsequent anyon beam.

(ii) Conserved current for a single anyon: Next we derive the conserved probability current for the anyon $\partial^{\mu} j_{\mu}^{(s=1-\lambda)}=0$, where $j_{0}^{s}$ is the probability density. Since the anyon model of [13] is an extension of the spin 1 case we can take a cue from the latter where the conservation law $\partial^{\mu} j_{\mu}^{(s=1)}=0$ reads

$$
\begin{aligned}
\partial^{\mu} j_{\mu}^{(s=1)}= & \partial^{0}\left[F^{0 \dagger} F^{0}+F^{x \dagger} F^{x}+F^{y^{\dagger}} F^{y}\right] \\
& -\partial^{x}\left[F^{0 \dagger} F^{x}+F^{x \dagger} F^{0}\right]-\partial^{y}\left[F^{0 \dagger} F^{y}+F^{y^{\dagger}} F^{0}\right] \\
= & 0 .
\end{aligned}
$$

Considering the Fourier transform of the anyon equation of motion (5) in position space, a long calculation yields the conserved free (single) anyon current $j_{\mu}^{(1-\lambda)}$,

$$
\begin{aligned}
& \partial^{0} \sum_{n=0}^{\infty}\left[\left(f_{n}^{0 \dagger} f_{n}^{0}+f_{n}^{x \dagger} f_{n}^{x}+f_{n}^{y \dagger} f_{n}^{y}\right)\right. \\
& \left.\quad-i\left(f_{n}^{y \dagger} K_{n n^{\prime}}^{0} f_{n^{\prime}}^{x}-f_{n}^{x \dagger} K_{n n^{\prime}}^{0} f_{n^{\prime}}^{y}\right)\right] \\
& \quad-\partial^{x} \sum_{n=0}^{\infty}\left[\left(f_{n}^{0 \dagger} f_{n}^{x}+f_{n}^{x \dagger} f_{n}^{0}\right)-i\left(f_{n}^{y \dagger} K_{n n^{\prime}}^{x} f_{n^{\prime}}^{x}-f_{n}^{x \dagger} K_{n n^{\prime}}^{x} f_{n^{\prime}}^{y}\right)\right] \\
& \quad-\partial^{y} \sum_{n=0}^{\infty}\left[\left(f_{n}^{0 \dagger} f_{n}^{y}+f_{n}^{y \dagger} f_{n}^{0}\right)-i\left(f_{n}^{y \dagger} K_{n n^{\prime}}^{y} f_{n^{\prime}}^{x}-f_{n}^{x \dagger} K_{n n^{\prime}}^{y} f_{n^{\prime}}^{y}\right)\right] \\
& =0,
\end{aligned}
$$

where we have explicitly shown the summation over $n$, the anyonic index. For $\lambda=0$ the current $j_{\mu}^{(1-\lambda)}$ reduces to the spin 1 current $j_{\mu}^{(1)}$ of (8). A nontrivial check of the consistency of the expressions for the anyon current (9) is to substitute $f_{n}^{a}$ from (7) to yield

$$
\begin{aligned}
& j^{0}=(1-\lambda) E / m ; \quad j^{x}=(1-\lambda) p^{x} / m ; \\
& j^{y}=(1-\lambda) p^{y} / m \rightarrow j^{\mu}=(1-\lambda) \frac{p^{\mu}}{m}=s \frac{p^{\mu}}{m} .
\end{aligned}
$$

For computational details see Supplemental Material B [18].

(iii) Anyon wave packet: Our aim is to construct the anyon current, not for a single anyon as done above, but for an anyon wave packet that can be amenable to experimental verification. Let us now construct the anyon wave packet 
that we want to move towards, say, $+x$ direction. Since we have superposed plane waves, later figures reveal that the current density has a sharply peaked profile with the $y$ component of current density having a comparatively reduced value. Note an important difference in geometry between our construction and that of the three-dimensional vortex beam [2]. In the latter case the free monoenergetic plane wave solutions (to be superposed) are distributed over the surface of a right circular cone with identical momentum amplitude in the propagation direction. However, for our anyon wave packet, in a planar geometry the above is not possible. Instead we use the superposition scheme where the azimuthal angle $\phi$ of momenta of the plane wave is integrated symmetrically from $\phi_{0}=-\pi / 2$ to $\phi_{0}=+\pi / 2$. In Fig. 1 we have shown profiles for charge density of anyon beam, $J_{0}^{\lambda}$, for $\lambda=0.6 \rightarrow s=1-\lambda=0.4$ for $\phi_{0}= \pm \pi / 2$.

Hence, considering superpositions of anyon plane waves with fixed spin $s=1-\lambda$, fixed energy $E$ and fixed kinetic energy $p=\sqrt{\left(p^{x}\right)^{2}+\left(p^{y}\right)^{2}}$, for the special case of integration limits $\phi_{0}= \pm \pi / 2$, the superposition $F_{n}^{a}$ appears as
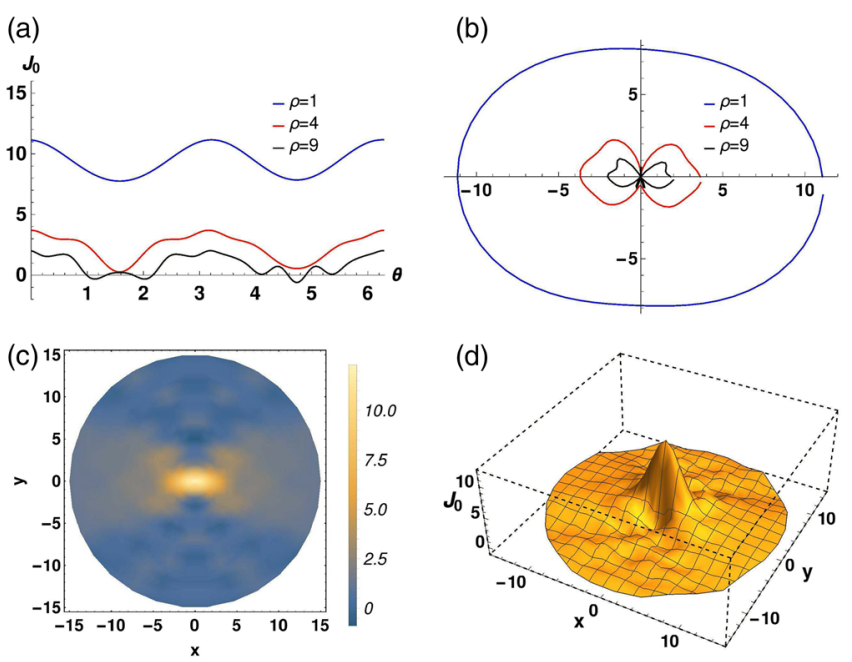

FIG. 1. (a) $J^{0}-\theta$ for different values of $\rho$. (b) Polar plot for $J^{0}$ with $\theta$ for fixed $\rho$ where the radial distance is the magnitude of $J^{0}$. (c) Density plot of $J^{0}$. (d) 3D plot of $J^{0}$. We set $\lambda=0.6$ and the integration ranges from $-\pi / 2$ to $+\pi / 2$.

$$
F_{n}^{a}(p, x)=\mathcal{A}_{n} \int_{-\pi / 2}^{\pi / 2}\left[\begin{array}{c}
\frac{p}{m} e^{i n \alpha} e^{i \alpha} \\
\left(1+\frac{p^{2}}{M} \cos \alpha e^{i \alpha}\right) e^{i n \alpha} \\
\left(i+\frac{p^{2}}{M} \sin \alpha e^{i \alpha}\right) e^{i n \alpha}
\end{array}\right]\left(e^{i(x \cos \alpha+y \sin \alpha)}+e^{i(x \cos \alpha-y \sin \alpha)}\right) d \alpha,
$$

where $\mathcal{A}_{n}=\frac{1}{2}(2 m / E+m)^{\lambda} \sqrt{[\Gamma(2 \lambda+n) / n ! \Gamma(2 \lambda)]} \sqrt{m / E}(p / E+m)^{n}, \quad x \equiv p x, \quad y \equiv p y$, and $M=m(E+m)$. The expression is symmetric separately under $x \rightarrow-x$ and $y \rightarrow-y$.

Conserved current for the anyon wave packet: The final analytical task is to substitute the anyon wave packet (11) in the expression of the anyon current (9). Since the current components are quadratic in the packet wave functions $F_{n}^{a}$, the final expressions are quite long and involved. We have shown only the expression for probability density $J^{0}$ and have relegated $J^{x}$ and $J^{y}$ to Supplemental Material C [18] together with a few computational steps. The cherished form of anyon beam probability density, in polar coordinates $x=\rho \cos \theta, y=\rho \sin \theta$, is

$$
\begin{aligned}
J^{0}(\rho, \theta)= & \left(\frac{2 m}{E+m}\right)^{2 \lambda} \frac{m}{E} \int_{-\pi / 2}^{\pi / 2} d \alpha \int_{-\pi / 2}^{\pi / 2} d \beta\left[e^{-2 i \rho p \sin [\theta-(\alpha+\beta) / 2] \sin [(\alpha-\beta) / 2]}+e^{-2 i \rho p\{\sin [\theta-(\alpha-\beta) / 2] \sin [(\alpha+\beta) / 2]}\right. \\
& \left.+e^{-2 i \rho p\{\sin [\theta+(\alpha-\beta) / 2]\} \sin -[(\alpha+\beta) / 2]}+e^{-2 i \rho p\{\sin [\theta+(\alpha-\beta) / 2]\} \sin [(-\alpha+\beta) / 2]}\right]\left[\left[1-e^{-i(\alpha-\beta)} \sigma^{2}\right]^{-2 \lambda}\right. \\
& \times\left\{\left(\frac{p}{2 m}\right)^{2} e^{-i(\alpha-\beta)}+\frac{1}{4}\left\{(1+\lambda)\left(2+\frac{2 p^{2}}{M}\right)+\frac{p^{4}}{M^{2}} e^{-i(\alpha-\beta)}[\cos (\alpha-\beta)+i \lambda \sin (\alpha-\beta)]\right\}\right\} \\
& \left.-\frac{\lambda}{2}\left\{\left[1-e^{-i(\alpha-\beta)} \sigma^{2}\right]^{-2 \lambda-1}\left(2+2 \frac{p^{2}}{M}+i \frac{p^{4}}{M^{2}} \sin (\alpha-\beta) e^{-i(\alpha-\beta)}\right)\right\}\right],
\end{aligned}
$$

where $\sigma=(p / E+m)$.

(iv) Visualizing the anyon beam: Unfortunately, closed form expressions for the anyon beam current components $J^{0}, J^{x}$, and $J^{x}$ are not possible to obtain. For different choices of $\lambda$ and $\phi_{0}$ the profiles of $J^{0}$ are given in Supplemental Material D [18]. Subsequently, in Figs. 2 and 3 , for the above values of $s, \phi_{0}$, we have plotted the profiles of $J^{a}, a \equiv x, y$ respectively, in three ways: a twodimensional plot of $J^{a}$ against the polar angle $\theta$ for a few values of the (planar) radial distance $\rho$, panel (a) in each group of figures. Another two-dimensional graph of the same data as panel (a) with magnitude of $J^{a}$ against $\theta$ for 
(a) $J_{x}$

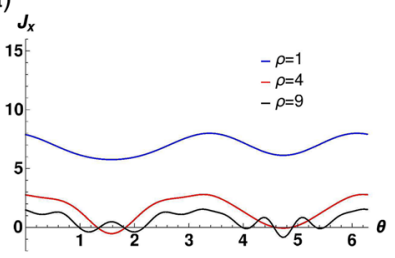

(c)

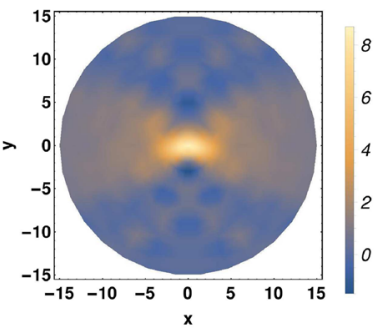

(b)

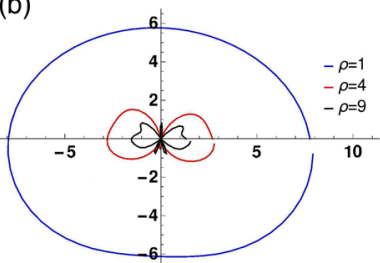

(d)

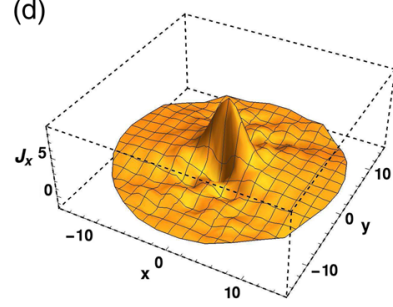

FIG. 2. $J^{x}(\lambda=0.6)$ with integration range $\pm \pi / 2$, where (a)-(d) correspond to the similar meaning as described in Fig. 1.

the same values of $\rho$ is shown in panel (b). A density plot in coordinate plane $x-y$ is given in panel (c). Finally, a threedimensional plot of $J^{a}$ in coordinate plane $x-y$ is provided in panel (d). Note that in panel (a), each continuous curve represents a fixed polar distance in coordinate plane $x-y$ with the height being a measure of $J^{a}$ whereas in panel (b), the radial distance is a measure of the intensity of $J^{a}$. Hence the curves that are farther away from the center in panel (b) represent points that are closer to the coordinate plane $x-y$.

As expected, all the wave packet profiles are symmetric about the abscissa since the packets are a superposition of plane waves that are symmetrically placed about the $x$ axis. Contrasting with the three-dimensional wave packets [2] it is clear that the planar anyon beams do not possess a vortex

(a)

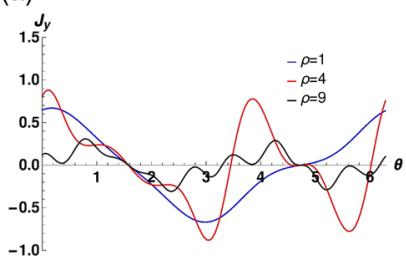

(c)

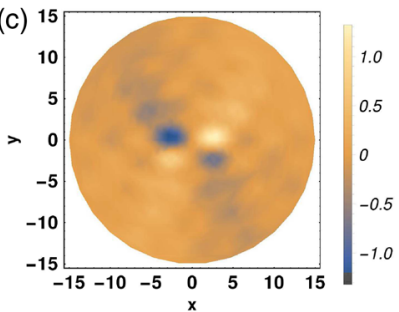

(b)

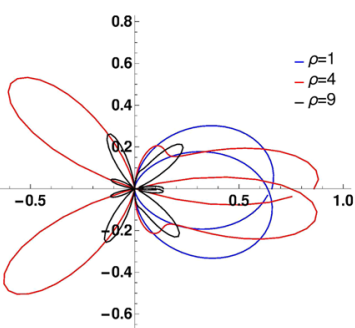

(d)

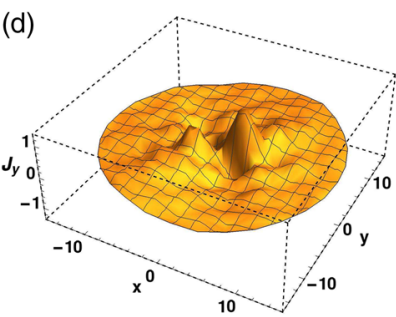

FIG. 3. $J^{y}(\lambda=0.6)$ with integration range $\pm \pi / 2$, where (a)-(d) represent a similar meaning as described in Fig. 1.

nature since the axial symmetry is manifestly broken while constructing a propagating anyon beam. This is also corroborated in the figures that do not have any destructive interference at the origin, a characteristic feature of vortex beams [2]. Hence the anyon beams are characterized by the spin value $s$ of the wave packet, which is the same as that of an individual plane wave single anyon component.

An important observation is that in the cases we have considered, $J^{0}$ is always positive, which has to be the case since it is the probability density. But $J^{x}$ is also positive throughout, whereas $J^{y}$ has positive and negative values in equal amount. Furthermore, maximum value of $J^{y}$ is far lower than each of $J^{0}$ and $J^{x}$. These reflect the nature of our construction of the anyon beam where all the plane waves have positive velocity along the $x$ direction but have pairwise opposite (both + ve and -ve) velocities along the $y$ direction. Hence, the anyon beam predominantly moves in the positive $x$ direction with the $y$ component effectively canceled out.

(v) Experimental possibilities: Anyons were detected in quantum antidot experiments [19] and in Laughlin quasiparticle interferometer [20]. In relation to simulation of high $T_{c}$ superconductivity by charged anyon fluid [21] their Josephson frequency has been observed [22] in 2D electrons in high magnetic field.

External electromagnetic field affects the anyon with charge $e$ and magnetic moment (see, e.g., [2])

$$
M=e \int d A\left(\epsilon_{i k} r^{i} j_{s}^{k}\right) / \int d A j_{s}^{0}
$$

for the single anyon current $j_{a}^{s}(8)$ using (7). We replace $j^{a}$ by $J^{a}$ using the wave packet (11). The nonstationary quantum superposition state (wave packet) can be created by exciting matter coherently with an ultrafast laser pulse, which is composed of eigenstates spanned by the frequency bandwidth of the laser [23].

The possibility of fault tolerant quantum computation by (non-Abelian) anyons has generated interest in controlled production of anyonic [12]: collective anyon excitations from electrons in the fractional quantum hall $(\mathrm{FQH})$ systems or from atoms in 1D optical lattices, creation of the $\mathrm{FQH}$ effect for photons [using one-dimensional (1D) or 2D cavity array], using a nonlinear resonator lattice subject to dynamic modulation for creating anyons from photons, among others.

Topologically ordered many-body states (with quenched kinetic energy) are generated with strongly interacting particles in magnetic field. The system minimizes interaction energy forming intricate patterns of long-range entanglement as observed in the FQH (semiconductor heterojunction, graphene [24,25], and van der Waals bilayers [26]). Formation of Laughlin states in synthetic quantum systems (ultracold atoms [27,28] and photons [2931]) has been developed. Recently in anyon optics, Laughlin states have been made out of photon pairs (in a synthetic 


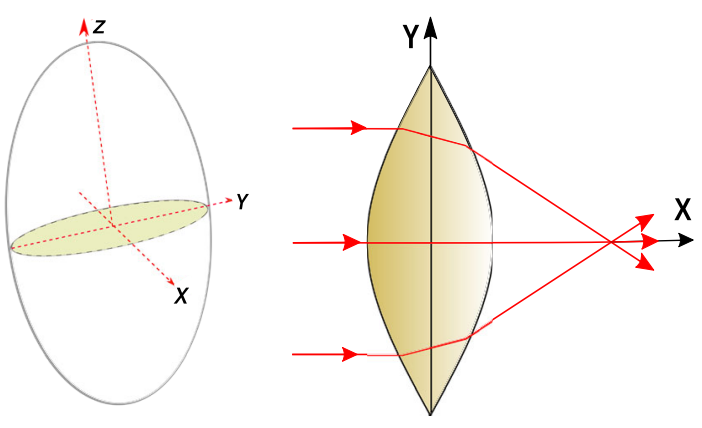

FIG. 4. Experimental setup: A slice (projection) of a 3D convex lens in the $x-y$ plane (left diagram) is taken out and shown separately as a thick lens in the right diagram. The red lines show the superposing wave vectors in the $x-y$ plane.

magnetic field for light induced from twisted optical cavity [32], strong photonic interactions via Rydberg atoms). These are modeled exploiting anyonic Hubbard Hamiltonian in ultracold-atom 1D lattices [33]. Anyon imaging with scanning tunneling microscope (STM) has also been achieved [33]. Theoretical [34,35] and experimental [36] studies for simulating anyonic NOON states with photons in waveguide lattices have appeared.

Our experimental proposal.-Following the work in [36], a two-photon NOON state with arbitrary anyonic symmetry is first prepared in a detuned directional coupler, and subsequently evolved in a Bloch oscillator emulated by a curved array. Then we use the planar analogue of a spiral phase plate where phase shift proportional to the path length of the waves passing through the plate occurs. Upon superposition this generates the anyon beam. This is schematically depicted in Fig. 4. The left panel of Fig. 4 shows a convex lens in three dimensions $x, y, z$ with parallel rays shown only along $x-y$ plane that converge on the $x$ axis. We consider an extremely thin slice of the lens in the $x$-y plane, which can be thought as the shaded area in the left panel. The same slice is drawn separately in the right panel that shows the planar superposition in our work.

(vi) Summary and future prospects: We have suggested the construction of relativistic anyon beam, a symmetrical superposition of Jackiw-Nair single anyon solutions. Explicit forms of wave packets, their significant features, and numerically plotted profiles of the anyon beam current are given. A laboratory model of the anyon beam construction is provided.

We thank Professor V. Parameswaran Nair for actively helping us in this project.

*santanu.maiti@isical.ac.in

[1] J. Durnin, J. Opt. Soc. Am. A 4, 651 (1987).

[2] K. Y. Bliokh, Y. P. Bliokh, S. Savel'ev, and F. Nori, Phys. Rev. Lett. 99, 190404 (2007); K. Y. Bliokh, M. R. Dennis, and F. Nori, Phys. Rev. Lett. 107, 174802 (2011).

[3] K. Y. Bliokh et al., Phys. Rep. 690, 1 (2017).
[4] L. Allen, M. W. Beijersbergen, R. J. C. Spreeuw, and J. P. Woerdman, Phys. Rev. A 45, 8185 (1992).

[5] A. J. Silenko, P. Zhang, and L. Zou, Phys. Rev. Lett. 121, 043202 (2018).

[6] F. Wilczek, Phys. Rev. Lett. 49, 957 (1982); see also Fractional Statistics and Anyon Superconductivity, edited by F. Wilczek (World Scientific, Singapore, 1990).

[7] J. M. Leinaas and J. Myrheim, Nuovo Cimento B 37, 1 (1977).

[8] Y. Zhang, Y.-W. Tan, H. L. Stormer, and P. Kim, Nature (London) 438, 201 (2005).

[9] A. A. Zibrov, C. Kometter, H. Zhou, E. M. Spanton, T. Taniguchi, K. Watanabe, M. P. Zaletel, and A. F. Young, Nature (London) 549, 360 (2017).

[10] A. Yu. Kitaev, Ann. Phys. (Amsterdam) 303, 2 (2003).

[11] H. Yao and S. A. Kivelson, Phys. Rev. Lett. 99, 247203 (2007); M. Kapfer, P. Roulleau, M. Santin, I. Farrer, D. A. Ritchie, and D. C. Glattli, Science 363, 846 (2019).

[12] L. Savary and L. Balents, Rep. Prog. Phys. 80, 016502 (2017); S. Dutta and E. J. Mueller, Phys. Rev. A 97, 033825 (2018).

[13] R. Jackiw and V. P. Nair, Phys. Rev. D 43, 1933 (1991).

[14] B. Binegar, J. Math. Phys. (N.Y.) 23, 1511 (1982).

[15] A. Perelomov, Generalized Coherent States and Their Applications (Springer, Berlin, 1986).

[16] V. P. Nair, arXiv:1606.06407.

[17] B. G. Wybourne, Classical Groups for Physicists (Wiley, New York, 1974).

[18] See Supplemental Material at http://link.aps.org/ supplemental/10.1103/PhysRevLett.123.164801, for explicit form of the free anyon wavefunction, computational details, anyon beam current, and examples of current density profile.

[19] V. J. Goldman and B. Su, Science 267, 1010 (1995); V. J. Goldman, J. Liu, and A. Zaslavsky, Phys. Rev. B 71, 153303 (2005).

[20] F. E. Camino, W. Zhou, and V. J. Goldman, arXiv:cond-mat/ 0611443.

[21] Y. Hosotani, Int. J. Mod. Phys. B 07, 2219 (1993); B. Abdullaev, G. Ortiz, U. Rössler, M. Musakhanov, and A. Nakamura, Phys. Rev. B 68, 165105 (2003).

[22] L. Yuan, M. Xiao, S. Xu, and S. Fan, Phys. Rev. A 96, 043864 (2017).

[23] L. J. Krause, K. J. Schafer, M. Ben-Nun, and K. R. Wilson, Phys. Rev. Lett. 79, 4978 (1997).

[24] X. Du, I. Skachko, F. Duerr, A. Luican, and E. Y. Andrei, Nature (London) 462, 192 (2009).

[25] K. I. Bolotin, F. Ghahari, M. D. Shulman, H. L. Stormer, and P. Kim, Nature (London) 462, 196 (2009).

[26] E. M. Spanton, A. A. Zibrov, H. Zhou, T. Taniguchi, K. Watanabe, M. P. Zaletel, and A. F. Young Science 360, 62 (2018).

[27] I. Bloch, J. Dalibard, and W. Zwerger, Rev. Mod. Phys. 80, 885 (2008).

[28] N. R. Cooper, J. Dalibard, and I. B. Spielman, Rev. Mod. Phys. 91, 015005 (2019).

[29] R. Umucallar, M. Wouters, and I. Carusotto, Phys. Rev. A 89, 023803 (2014).

[30] I. Carusotto and C. Ciuti, Rev. Mod. Phys. 85, 299 (2013). 
[31] T. Ozawa et al., Rev. Mod. Phys. 91, 015006 (2019).

[32] C. Noh and D. G. Angelakis, Rep. Prog. Phys. 80, 016401 (2017).

[33] C. Yannouleas and U. Landman, Phys. Rev. A 100, 013605 (2019).
[34] Y. Bromberg, Y. Lahini, and Y. Silberberg, Phys. Rev. Lett. 105, 263604 (2010).

[35] S. Longhi and G. Della Valle, Opt. Lett. 37, 2160 (2012).

[36] M. Lebugle, M. Gräfe, R. Heilmann, A. Perez-Leija, S. Nolte, and A. Szameit, Nat. Commun. 6, 8273 (2015). 